05

\title{
Зондирование молекул метанола в атмосфере по спектрам комбинационного рассеяния света
}

\author{
(C) В.Е. Привалов ${ }^{1}$, В.Г. Шеманин ${ }^{2,3}$ \\ ${ }^{1}$ Санкт-Петербургский политехнический университет Петра Великого, \\ 194021 Санкт-Петербург, Россия \\ ${ }^{2}$ Филиал Белгородского государственного технологического университета им. В.Г. Шухова, \\ 308012 Новороссийск, Россия \\ ${ }^{3}$ Новороссийский политехнический институт (филиал) \\ Кубанского государственного технологического университета, \\ 353900 Новороссийск, Россия \\ e-mail: vaevpriv@yandex.ru
}

Поступила в редакцию 03.11.2020 г.

В окончательной редакции 04.04.2021 г.

Принята к публикации 05.04.2021 г.

Представлены результаты численного решения лидарного уравнения для комбинационного рассеяния света (КРС) молекулами метанола в направлении назад при зондировании молекул в атмосфере с концентрациями в диапазоне $10^{12}-10^{18} \mathrm{~cm}^{-3}$ на расстояниях до $1000 \mathrm{~m}$ в режиме синхронного счета фотонов. Показано, что за время измерения $1 \mathrm{~s}$ лидаром КРС на длине волны лазерного излучения $405 \mathrm{~nm}$ можно зондировать молекулы метанола с уровнем концентрации порядка $10^{16} \mathrm{~cm}^{-3}$ на расстояниях до $600 \mathrm{~m}$.

Ключевые слова: лидар, уравнение, комбинационное рассеяние света, метанол, концентрация, счет фотонов.

DOI: $10.21883 /$ OS.2021.08.51200.236-21

\section{Введение}

В настоящее время метанол все активнее используется в качестве замены традиционным видам моторного топлива [1]: он гораздо дешевле и при этом наносит гораздо меньший вред окружающей среде, чем привычные бензин, керосин или дизтопливо. Поэтому становится актуальной разработка новых лидаров для измерения концентрации молекул метанола $\mathrm{CH}_{3} \mathrm{OH}$ как загрязняющего вещества на уровне предельно-допустимых концентраций (ПДК) в атмосферном воздухе. Для метанола, класс опасности которого 3 , значение ПДК $1.0 \mathrm{mg} / \mathrm{m}^{3}[2,3]$ или порядка $1.9 \cdot 10^{16} \mathrm{~cm}^{-3}$.

Ранее было показано, что лидар комбинационного рассеяния света (КРС) может использоваться для дистанционного измерения концентрации молекул на уровне сотен ПДК и более $[2,4,5]$. А мониторинг паров метанола лидаром в атмосферном пограничном слое в диапазоне расстояний зондирования до $1500 \mathrm{~m}$ может стать эффективным способом решения этой проблемы. Поэтому целью настоящей работы является оценка параметров лидара КРС для зондирования молекул метанола в атмосферном пограничном слое на уровне ПДК и выше в режиме синхронного счета фотонов. Для этого выполнено численное решение лидарного уравнения для КРС молекулами метанола с целью выбора длины волны лазерного излучения и параметров лидара для измерения минимального значения концентрации молекул $\mathrm{CH}_{3} \mathrm{OH}$ на заданном расстоянии зондирования.

\section{Спектр КРС метанола}

Был выполнен анализ спектра КРС молекулами метанола для выбора сильных характеристических полос КРС метанола и сравнения их с полосами возможных молекул примесей, таких как метан, этанол и формальдегид в предположении, что концентрации исследуемых молекул равны 1 ПДК. Значение максимумов полос валентных СН-колебаний всех четырех молекул и валентных СО-колебаний молекул метанола, этанола и формальдегида взяты из [2,5-7] и собраны в табл. 1. Значения дифференциальных сечений КРС для исследуемых молекул взяты из [5,8] и оценены по данным [5] для сравнения интенсивностей полос. Эти параметры также собраны в табл. 1.

По данным табл. 1 был сделан вывод о том, что зондирование молекул метанола может быть реализовано по двум выбранным полосам валентных колебаний $\mathrm{CH}$ и СO.

\section{Лидар КРС}

Для регистрации спектра КРС рассмотрим оптическую схему варианта лидара из [2,9], приведенную на рис. 1.

В качестве возможных вариантов рассмотрим использование второй гармоники YAG:Nd-лазера с длиной волны $532 \mathrm{~nm}$ и полупроводниковых лазеров с длинами волн 405 и $655 \mathrm{~nm}$ с длительностью импульсов $10 \mathrm{~ns}$ и 
Таблица 1. Значения максимумов полос КРС валентных СН- и валентных СО-колебаний всех исследованных молекул

\begin{tabular}{c|c|c|c|c|c|c|c|c}
\hline Молекула & \multicolumn{2}{|c|}{$\mathrm{CH}_{3} \mathrm{OH}$} & \multicolumn{2}{c|}{$\mathrm{CH}_{4}$} & \multicolumn{2}{c|}{$\mathrm{C}_{2} \mathrm{H}_{5} \mathrm{OH}$} & \multicolumn{2}{c}{$\mathrm{CH}_{2} \mathrm{O}$} \\
\hline $\begin{array}{c}\text { Параметры } \\
\text { полос }\end{array}$ & $v \mathrm{CH}_{\text {sym }}$ & $v \mathrm{CO}$ & $v \mathrm{CH}_{\text {sym }}$ & $v \mathrm{CH}_{\mathrm{as}}$ & $v \mathrm{CH}_{\text {sym }}$ & $v \mathrm{CO}$ & $v \mathrm{CH}_{\text {sym }}$ & $v \mathrm{CO}^{-1}$ \\
\hline Максимум, cm & 2846 & 1033 & 2914 & 3017 & 2943 & 1079 & 2766 & 1746 \\
\hline $\begin{array}{c}\text { Дифференциальное } \\
\text { сечение, } 10^{30} \mathrm{~cm}^{2}\end{array}$ & 14 & & & & 19 & & & 3.8
\end{tabular}

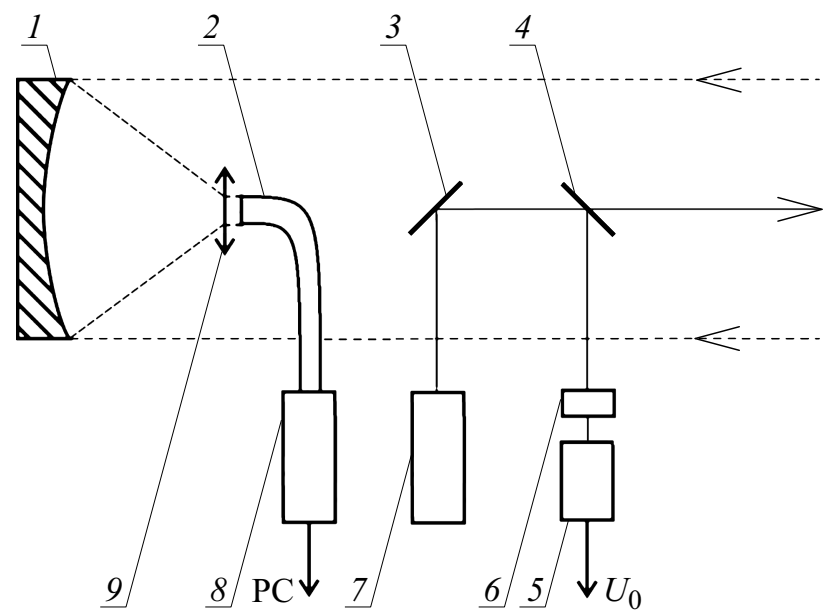

Рис. 1. Оптическая схема лидара КРС: 1 - сферическое зеркало приемного телескопа, 2 - волоконный ввод, 3 глухое зеркало, 4 - стеклянная пластина, 5 - интерференционный светофильтр, 6 - фотоприемник, 7 - лазер, 8 микроспектрометр, 9 - линзовый объектив.

энергиями в импульсе до $100 \mu \mathrm{J}$ при частоте следования лазерных импульсов $f$ до $1 \mathrm{MHz}[2]$.

Излучение лазера 7 направлялось вдоль оси приемного телескопа, а излучение КРС молекулами метанола в атмосферном пограничном слое в направлении назад собиралось приемным телескопом типа Ньютона со сферическим зеркалом 1 диаметром $400 \mathrm{~mm}$ и фокусировалось линзовым объективом 9 в волоконный световод 2 микро-спектрометра FSD-8 8 , работающего под управлением ПК. Часть лазерного излучения направлялось стеклянной пластиной 4 через интерференционный светофильтр 5 на фотоприемник 6 , напряжение $U_{0}$ с которого записывалось в память ПК для контроля энергии лазерного импульса и формирования начала отсчета времени измерения.

\section{Лидарное уравнение для КРС}

Лазер лидара будем характеризовать числом фотонов $n_{0}$ в импульсе посылаемого в атмосферу лазерного излучения длительностью $\tau_{0}$, а линию генерации лазера будем считать гауссовой с максимумом на частоте $v_{0}$ и полушириной $\Gamma_{0}$. Тогда число фотонов $n(v, z)$ излучения
КРС исследуемыми молекулами метанола с концентрацией $N(z)$ на фотоприемнике лидара можно получить из лидарного уравнения в направлении назад в режиме счета фотонов как интеграл в интервале от $\left(v_{0}-\Gamma_{0}\right)$ до $\left(v_{R}+\Gamma_{R}\right)[2,9,10]$ :

$$
\begin{aligned}
& n(v, z)=\frac{c \tau_{0}}{2 z^{0}} n_{0} S_{0} G(z) N(z) f t_{d}(d \sigma \Omega) \int_{v_{0}-\Gamma}^{v_{R}+\Gamma_{a}} \Phi\left(v^{\prime}\right) \\
& \times \exp \left[-\int_{0}^{z}\left\{\alpha\left(v_{0}, r\right)+\alpha\left(v_{R}, r\right)\right\} d r\right] A\left(v^{\prime}\right) d v^{\prime},
\end{aligned}
$$

где $n(v, z)$ - число фотонов, зарегистрированное фотодетектором лидара на частоте $v_{R}$ КРС с расстояния зондирования $z ; n_{0}$ - число фотонов на частоте $v_{0}$ лазерного излучения, которое определяется выражением $n_{0}=E_{0} / h v_{0}$, где $E_{0}-$ энергия импульса лазерного излучения длительностью $\tau_{0}$ (в отличие от $\tau_{1}-$ времени одного измерения или длительности синхроимпульса), $h$ - постоянная Планка. Кроме того, введены обозначения: $S_{0}$ - площадь приемной апертуры телескопа, $G(z)$ - геометрическая функция лидара [2,5], $0<G(z)<1$. А также $N(z)$ - концентрация исследуемых молекул, $t_{d}-$ время измерения или накопления сигнала, $(d \sigma / d \Omega)$ - дифференциальное сечение КРС исследуемыми молекулами; величины $\alpha\left(v_{0}, r\right)$ и $\alpha\left(v_{R}, r\right)$ - это коэффициенты ослабления на частотах лазерного излучения и КРС молекулами метанола, они характеризуют пропускание атмосферы $[2,11] ; \Phi(v)-$ функция Гаусса для распределения фотонов лазерного излучения в линии генерации [12]. Когда полуширина линии генерации много меньше, чем полуширина аппаратной функции лидара, можем считать, что $\Phi(v)=1$. Кроме того, будем считать, что аппаратная функция или спектральный коэффициент пропускания приемной системы лидара $A(v)[2,5,10]$ также описывается функцией Гаусса и настроена точно на частоту $v_{R}$ полосы КРС молекулами метанола с полушириной $\Gamma_{a}$, которая больше, чем $\Gamma_{R}$ и $\Gamma_{0}$ :

$$
A(v)=\frac{K_{1} \xi(v)}{\sqrt{2 \pi} \Gamma_{0}} \exp \left\{-\left(v-v_{R T}\right)^{2} /\left(2 \Gamma_{a}^{2}\right)\right\},
$$

где $K_{1}$ - лидарная константа на частоте $v_{0}$ лазерного излучения, измеренная экспериментально в [13]; 
$\xi\left(v_{R}\right)$ - относительная спектральная чувствительность фотоприемника на частоте $v_{R}$ излучения КРС. Предположение о том, что полуширина аппаратной функции на порядок больше, чем полуширина линии генерации лазера, вполне допустимо для промышленных лазеров и спектрометров [2]. Тогда перепишем уравнение (1) в виде

$$
n(v, z)=\frac{B}{z^{2}} N(z) t_{d} I(v, z)
$$

в котором константа $B$ есть

$$
B=\frac{c \tau_{0}}{2} S_{0} f
$$

и функция частоты и расстояния зондирования $I(v, z)$ имет вид

$$
\begin{aligned}
& I(\nu, z)=G(z) n_{0} \int_{\nu_{0}-\Gamma}^{v_{R}+\Gamma_{a}}(d \sigma / d \Omega) \times \\
& \times \exp \left[-\int_{0}^{z}\left\{\alpha\left(v_{0}, r\right)+\alpha\left(v_{R}, r\right)\right\} d r\right] A\left(v^{\prime}\right) d v^{\prime} .
\end{aligned}
$$

Сделаем некоторые преобразования, вынеся дифференциальное сечение КРС (оно выступает как молекулярная константа) и экспоненту из-под интеграла. Получим

$$
\begin{aligned}
& I(\nu, z)=G(z) n_{0}(d \sigma / d \Omega) \times \\
& \times \exp \left[-\int_{0}^{z}\left\{\alpha\left(v_{0}, r\right)+\alpha\left(v_{R}, r\right)\right\} d r\right] \int_{v_{0}-\Gamma}^{v_{R}+\Gamma_{a}} A\left(v^{\prime}\right) d v^{\prime} .
\end{aligned}
$$

Последний интеграл может быть преобразован с учетом интеграла ошибок [10] в произведение

$$
\begin{aligned}
& \int_{\nu_{0}-\Gamma}^{v_{R}+\Gamma_{a}} A\left(v^{\prime}\right) d v^{\prime}=\frac{K_{1} \xi(v)}{\sqrt{2 \pi} \Gamma_{a}} \int_{\nu_{0}-\Gamma}^{v_{R}+\Gamma_{a}^{\prime}} \exp \left[-\left(v-v_{R}\right)^{2} /\left(2 \Gamma_{a}^{2}\right)\right] d v \\
& =\operatorname{erf}(1 / \sqrt{2}) K_{1} \xi(v)=\frac{K_{1} \xi(v)}{2} 0.68269=0.34 K_{1} \xi(v) .
\end{aligned}
$$

Тогда выражение (6) с учетом (7) перепишем в виде

$$
\begin{aligned}
I(v, z)= & G(z) n_{0}(d \sigma / d \Omega) 0.34 K_{1} \xi(v) \times \\
& \times \exp \left[-\int_{0}^{z}\left\{\alpha\left(v_{0}, r\right)+\alpha\left(v_{R}, r\right)\right\} d r\right] .
\end{aligned}
$$

Теперь с учетом (4) и (8) из уравнения (3) выразим концентрацию $N(z)$ :

$$
N(z)=\frac{n(v, z) z^{2}}{B t_{d} I(v, z)} .
$$

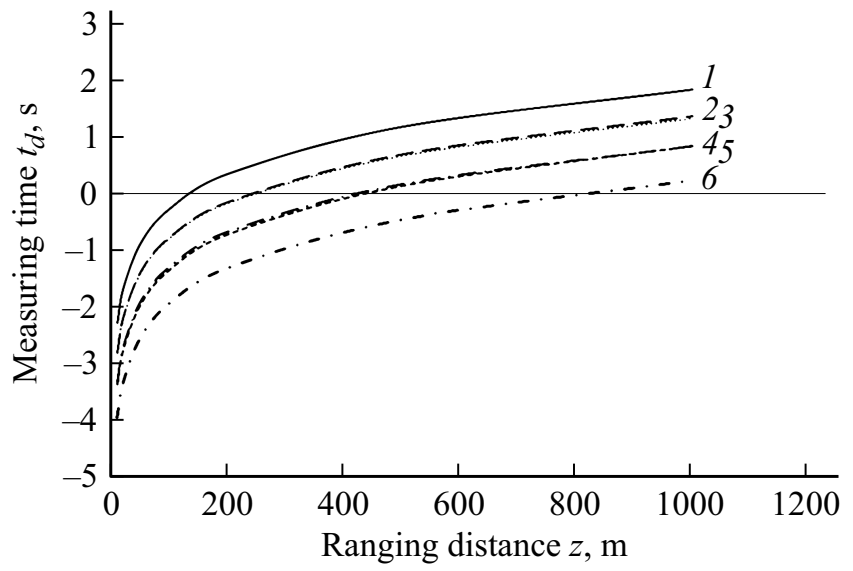

Рис. 2. Графики логарифмической зависимости времени измерения $t_{d}$ (в $\mathrm{s}$ ) от расстояния зондирования $z$ (в $\mathrm{m}$ ) для концентрации метанола $N(z)=10^{16} \mathrm{~cm}^{-3}$. Подробности в тексте.

\section{Результаты решения лидарного уравнения и обсуждение}

Рассмотрим однократное КРС и однородную атмосферу $[2,5,12]$. Значения максимумов полос валентных симметричных $\mathrm{CH}-$ и валентных СО-колебаний молекулы $\mathrm{CH}_{3} \mathrm{OH}$ взяты из [2,6-8] и равны 2846 и $1033 \mathrm{~cm}^{-1}$. Значения дифференциального сечения полос КРС молекулы метанола $(d \sigma / d \Omega)$ для выбранных длин волн лазерного излучения 405, 532 и $655 \mathrm{~nm}$ были оценены по данным [5,8], которые представлены в последней строке табл. 2. Рассчитанные с учетом зависимости $1 / \lambda^{4}$ (как в [4]) значения для интересующих нас длин волн приведены в табл. 2. Значения коэффициентов ослабления для этих частот $\alpha\left(v_{0}, z\right)$ и $\alpha\left(v_{R}, z\right)$ из $[13,14]$ также собраны в табл. 2. Поперечное сечение зеркала приемного телескопа лидара было $0.12 \mathrm{~m}^{2}$, а шаг по расстоянию - $7.5 \mathrm{~m}$ для времени одного измерения $50 \mathrm{~ns}$, за которое может быть зарегистрировано 200 фотонов. Лидарная константа $K_{1}$ определялась как произведение спектрального пропускания приемного телескопа на величину относительной спектральной чувствительности фотоприемника $\xi(\lambda)$ на длине волны $\lambda[2,13]$ из табл. 2.

На первом этапе построим зависимость времени измерения $t_{d}$ как меру эффективности нашего варианта лидара от расстояния зондирования $z$ для концентрации молекул $N(z)=10^{16} \mathrm{~cm}^{-3}$ и заданных параметров лидара. Рассмотрим численное решение уравнения (9) в предположении, что $G(z)$ для нашего случая равно 1 и за время измерения будут зарегистрированы 200 фотонов. Результаты решения уравнения (9) для этого случая представлены на рис. 2.

На рис. 2 представлены результаты для длин волн лазерного излучения 655 ( 1 и 3 ), 532 (2 и 4) и 405 (5 и 6) nm, причем кривые 2 и 3 и кривые 4 и 5 практически совпадают. Расчеты выполнены для полос симметричного валентного СН-колебания (3,4 и 6) и 
Таблица 2. Длины волн лазерного излучения и полос КРС молекул метанола, дифференциальные сечения колебательного КРС для двух полос, коэффициенты ослабления в атмосфере на этих длинах волн и относительная спектральная чувствительность фотоприемника

\begin{tabular}{|c|c|c|c|c|c|}
\hline \multirow{2}{*}{$\begin{array}{l}\text { Длины волн, } \\
\mathrm{nm}\end{array}$} & \multicolumn{2}{|c|}{$\begin{array}{c}\text { Дифференциальное сечение КРС } \\
(d \sigma / d \Omega) 10^{30}, \mathrm{~cm}^{2}\end{array}$} & \multicolumn{2}{|c|}{ Коэффициент ослабления } & \multirow{2}{*}{$\begin{array}{c}\text { Относительная спектральная } \\
\text { чувствительность ФП } \\
\xi(\lambda \\
\end{array}$} \\
\hline & CO $1033 \mathrm{~cm}^{-1}$ & $\mathrm{CH}_{\mathrm{sym}} 2846 \mathrm{~cm}^{-1}$ & $\alpha_{a}\left(\lambda_{0}, z\right), \mathrm{km}^{-1}$ & $\alpha_{a}(\lambda, z), \mathrm{km}^{-1}$ & \\
\hline $\begin{array}{c}655 \\
703 \\
805 \\
532 \\
563 \\
627 \\
405 \\
423 \\
458 \\
337.1\end{array}$ & 0.61 & $\begin{array}{l}2.26 \\
6.72 \\
14.0\end{array}$ & 0.14 & $\begin{array}{c}0.11 \\
0.106 \\
\\
0.15 \\
0.136 \\
\\
0.21 \\
0.173\end{array}$ & $\begin{array}{c}0.28 \\
0.25 \\
\\
0.48 \\
0.42 \\
\\
0.8 \\
0.84\end{array}$ \\
\hline
\end{tabular}

валентного СО-колебания (1,2 и 5) молекулы метанола. Как следует из графиков рис. 2, спектральные зависимости величин, входящих в уравнение (9), приводят к минимальному значению времени измерения концентрации исследуемых молекул $N(z)=10^{16} \mathrm{~cm}^{-3}$ на всех расстояниях зондирования для полосы симметричного валентного СН-колебания молекулы $\mathrm{CH}_{3} \mathrm{OH}$ (кривые 3,4 и 6) и увеличению этого значения для полосы валентного СО-колебания (1,2 и 7) для каждой длины волны лазерного излучения, причем на первых $100 \mathrm{~m}$ это значение растет: $11.7 \rightarrow 51.2 \rightarrow 163 \mathrm{~ms}$.

Минимальное время измерения можно получить на длине волны $405 \mathrm{~nm}$ : для $50 \mathrm{~m}$ это значение составит $2.86 \mathrm{~ms}$, для $100 \mathrm{~m}-11.7 \mathrm{~ms}$, для $500 \mathrm{~m}-345 \mathrm{~ms}$ и для $1 \mathrm{~km}-1.7 \mathrm{~s}$ при частоте следования лазерных импульсов $1 \mathrm{MHz}$ и времени одного измерения $50 \mathrm{~ns}$ (шаг по высоте $\Delta H=7.5 \mathrm{~m}$ ). Очевидно, что уменьшение времени измерения возможно за счет увеличения частоты следования лазерных импульсов или увеличения их энергии.

Теперь получим зависимость измеренной лидаром концентрации исследуемых молекул от расстояния зондирования $z$ для времени измерения $t_{d}=1 \mathrm{~s}$.

На рис. 3 расчеты выполнены для длин волн лазерного излучения 655 (1 и 3), 532 (2 и 4) и 405 (5 и 7) nm, причем кривые 2 и 3 и кривые 4 и 5 практически совпадают. Так же, как и ранее, представлены результаты для полос симметричного валентного СНколебания $(3,4$ и 6$)$ и валентного СО-колебания $(1,2$ и 5) молекулы метанола. На рис. 3 приведены результаты решения уравнения (9) для концентрации молекул метанола от $10^{12}$ до $10^{18} \mathrm{~cm}^{-3}$ и диапазона расстояний зондирования до $1000 \mathrm{~m}$ и времени измерения $1 \mathrm{~s}$. Сравнение с уровнем ПДК (прямая 6) показывает, что только использование лазерного излучения с длиной волны $405 \mathrm{~nm}$ позволит зарегистрировать такую концентрацию на расстоянии зондирования до $1 \mathrm{~km}$ при

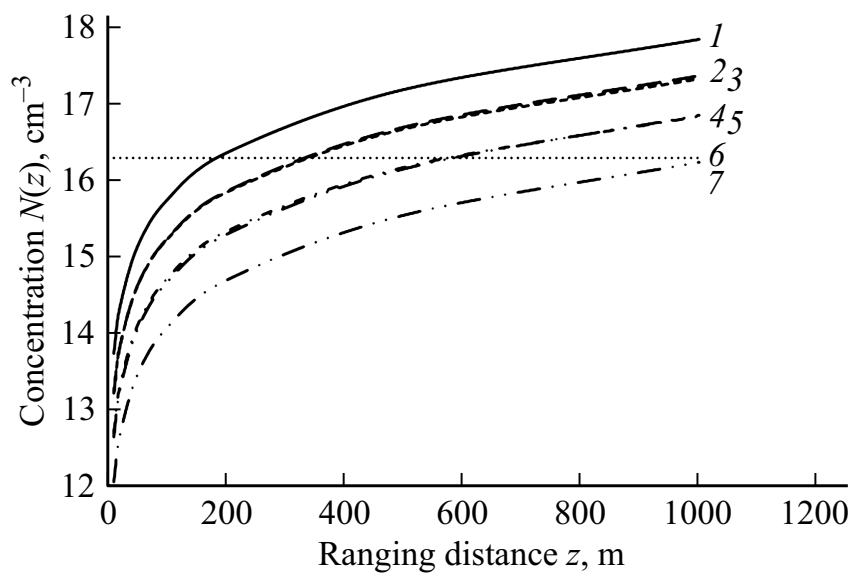

Рис. 3. Графики логарифмической зависимости концентрации $N(z)\left(\right.$ в $\left.\mathrm{cm}^{-3}\right)$ от расстояния зондирования $z$ (в $\left.\mathrm{m}\right)$. Прямая 6 - это уровень ПДК $1.9 \cdot 10^{16} \mathrm{~cm}^{-3}$. Подробности в тексте.

зондировании в полосе симметричного валентного СНколебания молекулы метанола, по полосе валентного СО-колебания - только до $600 \mathrm{~m}$.

\section{Выводы}

Таким образом, анализ полученных результатов показывает, что за время измерения $1 \mathrm{~s}$ лидаром КРС c выбранными параметрами на длине волны лазерного излучения $405 \mathrm{~nm}$ можно зондировать молекулы метанола с уровнем концентрации порядка $10^{16} \mathrm{~cm}^{-3}$ на расстояниях до $600 \mathrm{~m}$. Полученные результаты хорошо согласуются с данными [5] и отражают различие в уровнях ПДК исследованных молекул углеводородов [2], а разработка новых вариантов лидаров, например [15], открывает потенциальные возможности для дистанцион- 
ного зондирования низких концентраций молекул метанола в атмосфере над промышленным предприятием.

\section{Финансирование работы}

Работа была частично поддержана грантами РФФИ, проект № 9-42-230004 и проект № 19-45-230009.

\section{Конфликт интересов}

Авторы заявляют, что у них нет конфликта интересов.

\section{Список литературы}

[1] Новый вид топлива сулит России большие прибыли. 17 февраля 2020. [Электронный ресурс] Режим доступа: https://yandex.ru/turbo?promo=navbar\&utm_referrer

$=\mathrm{https} \% 3 \mathrm{~A} \% 2 \mathrm{~F} \% 2$ Fzen. yandex.com\&utm_campaign $=\mathrm{dbr} \&$ text $=$ https $\% 3 \mathrm{~A} \% 2 \mathrm{~F} \% 2 \mathrm{Fvz.ru} \% 2 \mathrm{Fnews} \%$ 2F $2020 \% 2 \mathrm{~F} 2 \% 2 \mathrm{~F} 17 \% 2 \mathrm{~F} 1024248 . \mathrm{html}$

[2] Привалов В.Е., Фотиади А.Э., Шеманин В.Г. Лазеры и экологический мониторинг атмосферы. СПб.: Лань, 2013. $288 \mathrm{c}$.

[3] Гигиенические нормативы ГН 2.1.6.3492-17 (с изменениями на 31 мая 2018 г.). Предельно допустимые концентрации (ПДК) загрязняющих веществ в атмосферном воздухе городских и сельских поселений.

[4] Креков Г.М., Крекова М.М., Суханов А.Я., Лысенко А.А. // Письма в ЖТФ. 2009. Т. 35. В. 15. С. 8.

[5] Межерис Р. Лазерное дистанционное зондирование. М.: Мир, 1987. $550 \mathrm{c}$.

[6] Свердлов Л.М., Ковнер М.А., Крайнов Е.П. Колебательные спектры многоатомных молекул. М.: Наука, 1970.

[7] Ismail Hakki Boyaci, Hüseyin Efe Genis, Burcu Guven, Ugur Tamerb, Neslihan Alperc. // J. Raman Spectrosc. 2012. V. 43. P. 1171. doi $10.1002 /$ jrs.3159

[8] Лазерный контроль атмосферы / Под. ред. Хинкли Э.Д. М.: Мир, 1979

[9] Привалов В.Е., Шеманин В.Г. // Измерительная техника. 2016. № 9. C. 22.

[10] Привалов В.Е., Шеманин В.Г. // Изв. РАН. Серия физическая. 2015. Т. 79. В. 2. С. 170.

[11] Донченко В.А., Кабанов М.В., Кауль Б.В., Самохвалов И.В. Атмосферная электрооптика. Томск: Изд-во НТЛ, 2010. С. 178-181.

[12] Долгих Г.И., Привалов В.Е. Лазеры. Лазерные системы. Владивосток: Изд. Дальнаука, 2009. 202 с.

[13] Привалов В.Е., Шеманин В.Г. Параметры лидаров для дистанционного зондирования газовых молекул и аэрозоля в атмосфере. СПб.: Балт. ГТУ, ВОЕНМЕХ, 2001. 57 с.

[14] Справочник по лазерам. / Под ред. Прохорова А.М. Т. I. М.: Советское Радио, 1978. 504 c.

[15] Привалов В.Е., Шеманин В.Г. // Опт. и спектр. 2018. T. 125. B. 4. C. 568. doi 10.21883/OS.2018.10.46714.129-18 